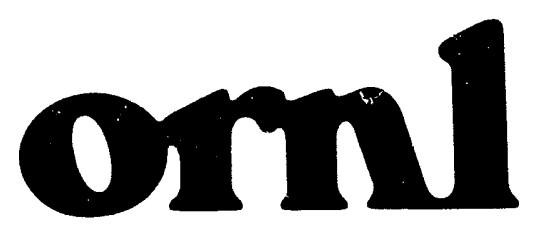

OAK RIDGE NATIONAL LABORATORY

MARTIN MARIETTA
The Significance of the Centripetal Acceleration Due to the Earth's Rotation on the Generation of Oceanic Circulation

\author{
R. P. Wichner
}


This report has been reproduced directly from the best avallable copy.

Available to DOE and DOE contractors from the Office of Scientific and Technical Information, P.O. Box 62, Oak Ridge, TN 37831; prices available from ;615) 576-8401, FTS 626-8401.

Available to the public from the National Technical Information Service, U.S. Department of Commerce, 5285 Port Royal Rd., Springfield, VA 22161.

This report was prepared as an account of work sponsored by an agancy of the United States Government. Neither the United States Government Iror any agency thereof, nor any of their employees, makes any warranty, express or implied, or assumes any legal liability or responsibitity for the accuracy, completeness, or usefulness of any information, apparatus, procuct, or process disclosed, or represents that its use would not infringe privatehy owned rights. Reference herein to any specific commercial product, process, or service ty trade name, trademark, manufacturer, or otherwise, does not necessarily constitute or imply its endorsement, recommendation, or favoring by the United States Government or any agency thereof. The views and opinions of authors expressed herein do not necessarily state or reflect those of the United States Government or any agency thereof. 


\section{Engineering Technology Division}

\section{THE SIGNIFICANCE OF THE CENTRIPETAL ACCELERATION DUE TO THE EARTH'S ROTATION ON THE GENERATION OF OCEANIC CIRCULATION}

R. P. Wichner

DATE PUBLISHED: NOVEMBER 1991

Prepared by the OAK RIDGE NATIONAL LABORATORY

Oak Ridge, Tennessee 37831-6285 managed by MARTIN MARIETTA ENERGY SYSTEMS, INC. for the U.S. DEPARTMENT OF ENERGY under contract DE-AC05-84OR21400 
CONTENTS

Page

LIST OF FIGURES $\ldots \ldots \ldots \ldots \ldots \ldots \ldots \ldots \ldots \ldots \ldots \ldots \ldots \ldots, \quad \mathbf{v}$

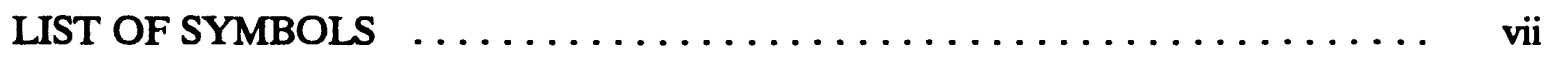

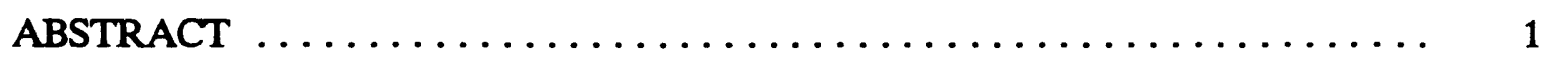

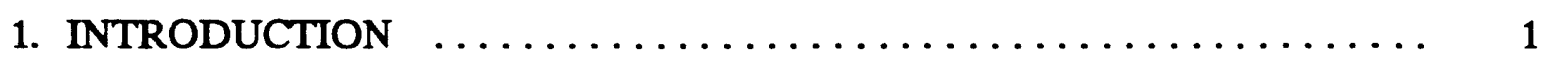

2. COMPARISON OF ACCELERATION DUE TO THE EARTH'S REVOLUTION AND ROTATION $\ldots \ldots \ldots \ldots \ldots \ldots \ldots \ldots \ldots, 2$

3. CIRCULATION MODEL CONCEPT BASED ON CENTRIFUGAL FORCE 5

4. CIRCULATION MODEL TEST $\ldots \ldots \ldots \ldots \ldots \ldots \ldots \ldots \ldots \ldots \ldots \ldots \ldots$

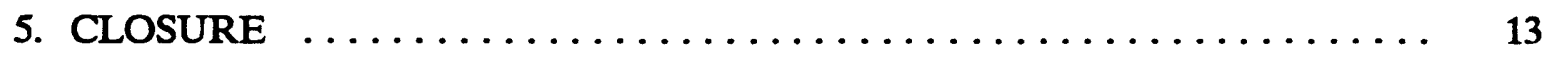

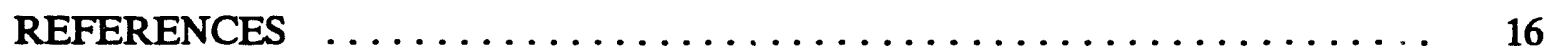




\section{LIST OF FIGURES}

Figure

Page

1 Normal and tangential components of centrifuga! body force in liquid held on rotating sphere $\ldots \ldots \ldots \ldots \ldots \ldots \ldots \ldots \ldots \ldots \ldots \ldots \ldots$

2 Formation of convective ceils in horizonal pool of liquid heated from

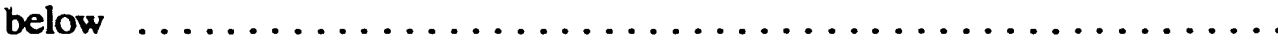

3 Ocean circulation pattern illustrating analogy to convection cells in pool heated from below

4 Idealized oceanic circuiation flow path $\ldots \ldots \ldots \ldots \ldots \ldots \ldots$ 


\section{SYMBOLS}
A cross-sectional area of flow
ap acceleration of fluid element in inertial reference frame
$a_{0}^{\prime}$ acceleration of nonrotating coordinate frame fixed to the earth
$a_{c e}$ acceleration due to earth's rotation
$a_{\infty}$ Coriolis acceleration
$a_{p}^{*}$ acceleration of fluid element as viewed on earth
F net force for circulation, Eq. (10)
$F_{c e}$ body force due to $a_{c e}$, with tangential and normal components $F_{c e, t}$ and $F_{c e, n}$
$F_{f}$ force vector on fluid element due to velocity gradients, Eq. (1)
$F_{R}$ force due to fluid resistance to circulation
D depth of wind-affected layer, Eq. (4)
g gravity acceleration of gravity on earth's surface
h depth of pool, Eq. (7)
$\mathrm{H}$ distance from equator to pole
$\mathrm{K}$ flow friction parameter; number of velocity heads, Eq. (13)
P pressure
$r_{e}$ radius vector from earth's center to surface, magnitude is $\mathbf{R}$
$\mathrm{Ra}_{\text {. }}$ Rayleigh stability parameter for a pool, Eq. (7)
Ra Rayleigh stability parameter for the ocean, Eq. (8)
$\mathrm{U}$ mean current velocity
v velocity of liquid volume element as observed on earth
$\alpha \quad$ thermal diffusivity of water
$\beta$ thermal expansion coefficient of water
$\theta$ latitude
$\nu \quad$ kinematic viscosity of water
$\rho$ water density
$\tau$ wind shear stress
$\omega_{c}$ rotation rate vector of earth 


\title{
THE SIGNIFICANCE OF THE CENTRIPETAL ACCELERATION DUE TO THE EARTH'S ROTATION ON THE GENERATION OF OCEANIC CIRCULATION
}

\begin{abstract}
R. P. Wichner
ABSTRACT

This report proposes that the tangential component of the centrifugal body force due to the earth's rotation plays a significant role as a motive force for the major oceanic circulations. A comparison of its magnitude relative to the Coriolis force and wind shear, on which current circulation models are based, indicates its potential effect is significant if an appropriate mechanism can be constructed that generates a circulation force. Such a mechanism is proposed, based on the coupled effect of water-density variations with the tangential component of the centrifugal force. An order-of-magnitude model, which equates the generated circulation force with a rough estimate of the flow resistance, indicates a favorable comparison between predicted and observed current velocity.
\end{abstract}

\section{INTRODUCTION}

While a general consensus exists that the major oceanic circulations are driven primarily by wind shear coupled with an effect due to the Coriolis force, on occasion comments in the literature appear which indicate that there is some doubt as to whether this indeed presents a complete and accurate picture. For example, Holland (1977) states that, "Despite substantial progress during the past few decades in understanding the large scale oceanic circulation, the description of the general circulation of the ocean is far from complete." A similar view has been more recently given by Dewar and Bane (1989), "We are still at the stage in our examination ... where the simplest classes of models ... need to be compared with data." The models alluded to are those developed in the 1950 s by Stommel, Munk, and Fofonoff. Although there has been an explosion of computational capability, the basic modeling assumptions appear not to have changed significantly in recent years. Indeed, a recent computation of oceanic circulation (Holland and Williams 1987) is based on a model by Bryan (1969) that is a computational adaptation of concepts presented by Fofonoff (1962), Eckart (1962), and others. 
These models consist of a set of equations that include a fluid force balance, a fluid conservation equation, and an enthalpy and a salinity balance. Typically, the force balance is written on a volume element containing a unit mass of fluid:

$$
\left(\frac{\partial \nabla}{\partial t}+(\nabla) \cdot \nabla \nabla\right)+2 \omega_{e} \times \nabla=-\frac{\nabla p}{\rho}+F_{f}+g
$$

The term in parentheses is the acceleration of the element as viewed from the surface of the earth (see list of symbols). The second term is the Coriolis acceleration, which at least partially accounts for the fact that in earth-fixed coordinate system, in which all the vectors in Eq. (1) are defined, is not an inertial frame as required for Newton's law. For completeness, other acceleration terms are required, which are usually neglected. Equation (1) is, of course, an expression of Newton's law with accelerations on the left responding to forces on the right.

Other simplifications not directly germane here are generally applied. However, it should be noted that solution methods for Eq. (1) emphasize force elements parallel to the surface of the earth as being far more significant for generating currents compared with components perpendicular to the surface. The Coriolis acceleration may have both parallel and perpendicular components, depending on the direction of $v$. The perpendicular components are generally neglected, as well as the perpendicular components of the fluid stress vector $\mathbf{F}$.

\section{COMPARISON OF ACCELERATIONS DUE TO THE EARTHS REVOLUTION AND ROTATION}

If one assumes a fixed reference frame centered in the sun to be the inertial frame, then it may be noted that Eq. (1) omits the centripetal accelerations due to the revolution of the earth about the sun and the rotation about its axis. The acceleration of the fluid element may be written as

$$
a_{p}=a_{0}^{\prime}+a_{\infty}+a_{\infty}+a_{, \prime}^{\prime \prime}
$$


where $\mathrm{a}_{\mathrm{p}}$ is the acceleration of the fluid element in the inertial frame. It is composed of the vector sum of $a_{b}^{\prime}$, the acceleration of a nonrotating frame centered at the earth relative to the sun; $a_{\infty e}$, the centripetal acceleration due to the earth's rotation; $a_{\infty 0}$, the Coriolis acceleration; and $a_{p}^{*}$, the acceleration of the fluid element as seen by an observer on earth. The vector $a_{p}^{*}$ is identical to the term in parentheses in Eq. (1). The accelerations $a_{b}^{\prime}$ and $a_{c e}$ should be included in the left side of Eq. (1) to render it a more closely valid expression of Newton's law.

This simplified view of coordinate frame accelerations neglects components due to the interaction of the earth-moon system. However, the principal purpose of the report is to examine the possible effect of body forces due to $a_{c e}$ on oceanic circulation. It is only intended here to call attention to the fact that several effective body force terms are generally omitted from oceanic circulation models and, in particular, that body forces due to $a_{c e}$ may play a significant role in defining the nature of the major ocean flows.

The acceleration of the coordinate frame fixed to the earth produces real enough forces as perceived on the earth's surface; for example, the centripetal acceleration a may properly be viewed as the centrifugal body force per unit mass $\mathbf{F}_{\propto \mathrm{c}}$ given by

$$
\boldsymbol{F}_{c \bullet}=-\boldsymbol{a}_{c_{0}}=-\omega_{0} \times\left(\omega_{0} \times \boldsymbol{x}_{0}\right)
$$

and included as a body force on the right side of Eq. (1). Similarly, the Coriolis and earth's revolution components of $a_{p}$ are perceived on earth as real body forces and could be included as such in the force balance.

Table 1 compares typical values of the magnitudes of $a_{b}^{\prime}, a_{c e}$, and $a_{c \infty}$. The vector $a_{0}^{\prime}$ is essentially constant on earth and thus attains maximum tangential values along the circumference lying in the plane normal to the vector from the sun to the earth. The magnitude listed for $a_{c e}$ refers to the component parallel to the earth's surface evaluated at $45^{\circ}$ latitude. The value of the Coriolis acceleration is based on a northward velocity of 0.5 $\mathrm{m} / \mathrm{s}$, which is approximately average for the Gulf Stream at this latitude. We note, perhaps surprisingly, that accelerations due to the revolution about the sun exceed that of a typical Coriolis acceleration, while the parallel component of the centripetal acceleration is significantly larger than both. However, such a direct comparison may be misleading because the means by which they affect flow differ. Accelerations $\mathbf{a}_{\mathbf{o}}^{\prime}$, $\mathbf{a}_{\mathrm{ce}}$, and, for that matter, $\mathbf{g}$ may 
Table 1. Comparison of effects due to the acceleration of the earth and effective acceleration due to wind shear

\begin{tabular}{|c|c|c|}
\hline Component & $\begin{array}{l}\text { Acceleration } \\
\qquad\left(\mathrm{m} / \mathrm{s}^{2}\right)\end{array}$ & $\begin{array}{c}\text { Density-corrected } \\
\text { effect } \\
\left(\mathrm{m} / \mathrm{s}^{2}\right)\end{array}$ \\
\hline $\begin{array}{l}a_{\circ}^{\prime} \quad \text { (revolution } \\
\text { about the sun) }\end{array}$ & $5.9 \times 10^{-3}$ & $1.8 \times 10^{-5}$ \\
\hline $\begin{array}{l}a_{c e} \text { (due to earth's } \\
\text { rotation) }\end{array}$ & $1.7 \times 10^{-2}$ & $5.0 \times 10^{-5}$ \\
\hline$a_{\infty}$ (Coriolis) $^{c}$ & $5.2 \times 10^{-5}$ & $5.2 \times 10^{-5}$ \\
\hline$a_{\text {sbear }}(\text { wind })^{d}$ & $5 \times 10^{-7}$ & $5 \times 10^{-7}$ \\
\hline
\end{tabular}

$a_{a}^{\prime}$ and $a_{c e}$ multiplied by typical $\Delta \rho / \rho$ of 0.003 .

bTangential component at $45^{\circ}$ latitude.

'Based on $0.5-\mathrm{m} / \mathrm{s}$ flow on longitude at $45^{\circ}$ latitude.

${ }^{\star}$ Based on 0.05-Pa shear and 100-m-deep wind-affected layer.

cause convective flow by means of density differences in the water, whereas the effects of the Coriolis acceleration and wind shear are more direct. Therefore, a fairer comparison of their relative effect on generating flow may be obtained by multiplying the magnitudes of $a_{0}^{\prime}$ and $a_{c e}$ by a typical fractional density difference, say 0.003 , which is the fractional density difference between pure water at 0 and $25^{\circ} \mathrm{C}$. These comparisons are shown in the third column of the table. Note that these assumptions lead to the observation that the potential effect of the centripetal acceleration due to the earth's rotation is comparable to the Coriolis force.

A rough comparison of the relative effect of wind shear and the body forces due to the acceleration of the earth's coordinate frame may be made by distributing the wind shear over the wind-affected depth to obtain an effective acceleration,

$$
a_{\text {man }}=\frac{\tau_{\text {mid }}}{\rho D}
$$

where $\tau$ is the wind shear and $D$ the depih of the surface-affected layer. The range of values presented by Boning et al. (1991) indicates that $0.05 \mathrm{~Pa}\left(0.5\right.$ dynes $\left./ \mathrm{cm}^{2}\right)$ is a reasonable 
estimate for a typical wind shear. If the depth of the wind-affected layer is assumed to be $100 \mathrm{~m}$, as suggested by Stewart (1969), then a typical, effective acceleration for wind shear of $5 \times 10^{-7} \mathrm{~m} / \mathrm{s}^{2}$ is obtained; this is significantly smaller than the accelerations due to the revolution and rotation of the earth.

The net meaning of this discussion is that if a reasonable model can be constructed for the generation of oceanic circulation by means of a centrifugal body force resulting from the earth's rotation, it would likely prove to be a significant addition to current models based on wind shear and Coriolis force effects.

\section{CIRCULATION MODEL CONCEPT BASED ON CENTRIFUGAL FORCE}

The centrifugal body force on a volume element of liquid held upon the surface of a rotating sphere is illustrated in Fig. 1. The magnitude and direction of $\mathbf{F}_{c e}$ (i.e., normal to the axis of rotation) are determined by Eq. (3). The magnitudes of tangential component $F_{c e, v}$ directed along a longitude toward the equator, and the normal component $F_{c e, n}$ are given by

$$
F_{c e, s}=\rho \omega_{e}^{2} R \cos \theta \sin \theta,
$$

and

$$
F_{c e, n}=\rho \omega_{e}^{2} R \cos ^{2} \theta
$$

The liquid surface assumes the shape of an oblate spheroid caused by the increase in the radial component of the centrifugal force with decreasing latitude.

Initially, a highly idealized system is assumed that would include an isothermal liquid that is stationary with respect to the rotating frame, a perfect sphere of radius $R$, no continental obstructions, and no surface tractive force due to wind. Such a system is in static equilibium with no driving force for the generation of flow. The Coriolis body force is absent, and the tangential component of the centrifugal force, which we will examine as a contributing factor in oceanic circulation, is balanced by the static pressure gradient due to the variation of 
ORNL-DWG 91M-2905 ETD

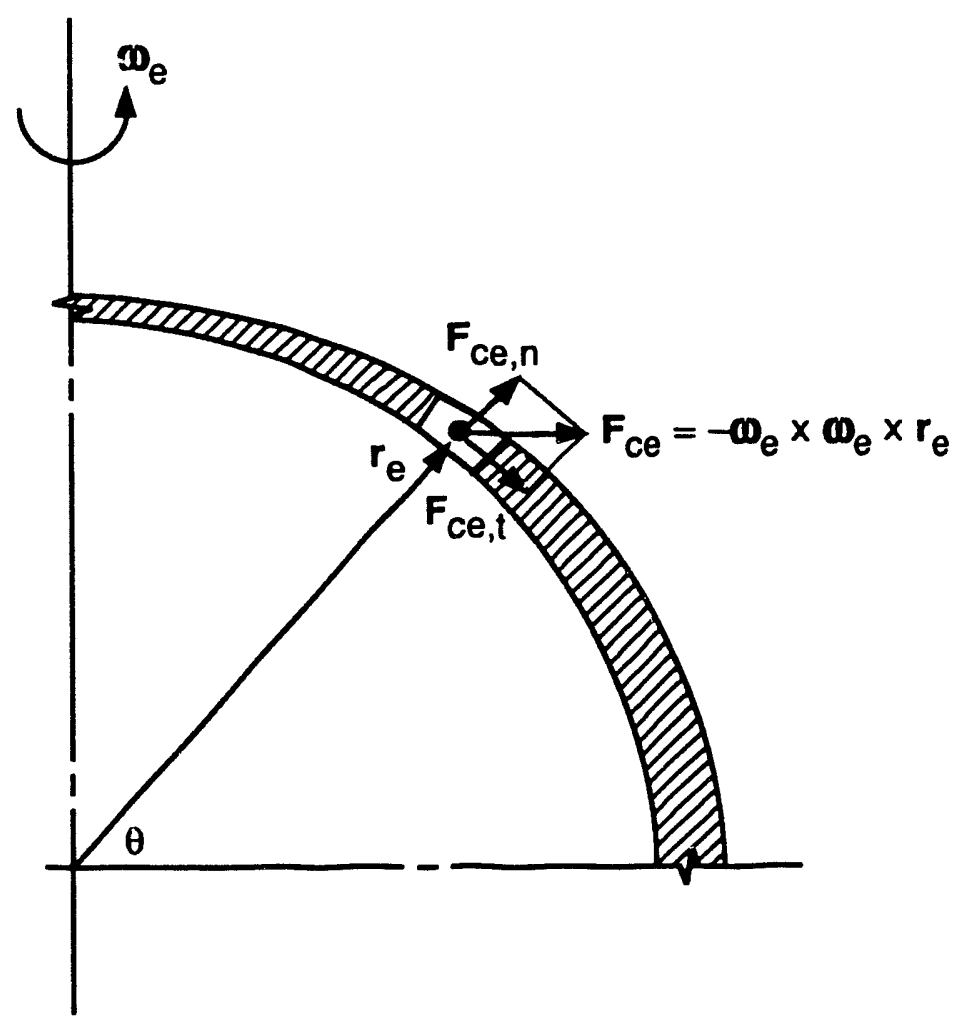

Fig. 1. Normal and tangential components of centrifugal body force in liquid held on rotating sphere.

depth with $\theta$. More important than force equilibrium, is that one senses that this systern is also stable, meaning that any sort of assumed small perturbation will decay with time. (This is stated here only intuitively.) However, staticaly balanced systems that prove to be unstable usually contain a self-evident "stress" that may be relieved by some dynamic process. The process may be the buckling of a column under compression, the generation of turbulence because of a need for a high degree of momentum transport imposed by boundary conditions, or the generation of convection currents in cases of high heat flux. No such stress is evident for the described isothermal system.

We now assume a liquid-density variation with latitude angle, $\rho(\theta)$, caused by higher insolation rates near the equator relative to the higher latitudes. In the absence of convective currents, heat would be transported from equatorial to polar regions by conduction in the liquid. Because the resistance to heat flow by means of molecular 
conduction is much higher than for even relatively slow convection, the temperature gradient with $\theta$ would, for this hypothetical situation, far exceed that which currently exists. The temperature variation with $\theta$ would be monotonically decreasing, and $\rho$ would be monotonically increasing with increasing latitude angle.

This report hypothesiszes that the effect of the increasing liquid density with latitude on the tangential component of the centrifugal acceleration is to create an instability that is relieved by the generation of the major oceanic circulations. That is, the condition of nonflow and heat transport from equator to pole by conduction is unstable relative to flow circulation and heat transport by means of convection. Moreover, it can be shown that the driving force for the circulation, which results from the effect of density variations on the tangential component of the centrifugal force, is consistent with the flow resistance of the observed circulation.

Visualization of the potential for such an instability may be aided by examining a roughly comparable situation, the classical case analyzed by Rayleigh (1916) of a shallow pool of liquid heated from below. Figure $2(a)$ illustrates the static situation, where heat is transferred by conduction from a source beneath the pool, creating a linear temperature gradient with depth. For modest temperature differences, the density variation with depth is approximately linear, and the resulting pressure variation is quadratic. Rayleigh showed that when the thermal and flow equations expressed for the nonflow case of Fig. 2(a) are perturbed, the perturbations grow with time into the circulatory system shown in Fig. 2(b)

ORNL-DWG 91M-2906 ETD

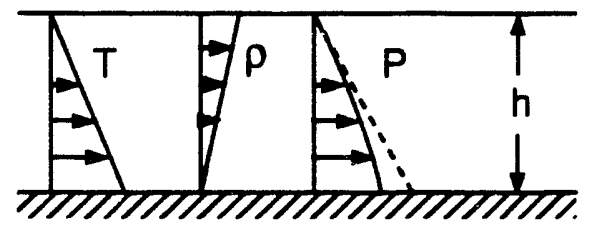

(a)
† HEAT

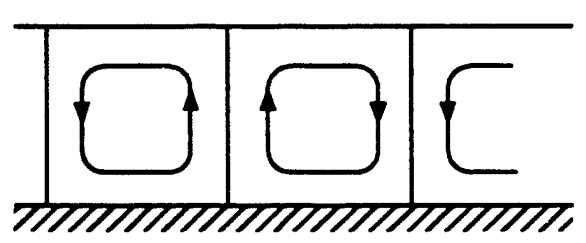

(b) $\uparrow$ HEAT

Fig. 2. Formation of convective cells in horizontal pool of liquid heated from below. ( $a$ ) Transfer of heat by conduction only and $(b)$ heat transfer primarily by means of convection cells. 
if the parameter $\mathrm{Ra}$, now termed the Rayleigh number, exceeds 1108 . The Rayleigh parameter for this situation is defined as

$$
R a_{h}=\frac{g \beta \Delta T h^{3}}{\alpha \nu} .
$$

According to a modern exposition (Bejan, 1984), Rayleigh was guided to the assumed form of the perturbation and to the final result by direct observation of the convection cells. In fact, although numerous flow instability analyses exist, only a few simple situations have been analyzed so thoroughly that the final, time-steady form of the instability is actually described theoretically.

An analogy relative to the case of liquid on a rotating sphere is obtained by recognizing that the tangential component of the centrifugal acceleration plays a role similar to that of the vertical pressure gradient in the heated pool. Figure 3 depicts the analogous situation. Note, the force component $F_{c a, t}$ acting above and below the equator generates fluid compression, and, similar to the heated pool, the region of lowest fluid density occurs in the area of highest compression.

We note that the tangential component of the centrifugal force provides a character common to all unstable situations, that of generating a "stress" with a potential for being relieved by some dynamic mechanism; that is, it creates a fluid compression in the tangential, north-south direction with a potential for fluid buckling, a mechanism often assumed to be a cause of eddy formation in a laminar flow field. For the isothermal situation, it was presumed without proof that the increase of liquid depth with decreasing latitude not only balanced forces but also created stability. For the nonisothermal case, we may write a Rayleigh number stability parameter analogous to the situation described in Fig. 2 by replacing the acceleration of gravity with its equivalent, the centrifugal force, and the pool depth with its equivalent, the length of the conduction path, the distance from equator to pole $\mathrm{H}$. That is, an oceanic, centrifugal-force Rayleigh number may be written as

$$
R a_{0}=\frac{\omega_{e}^{2} R \beta \Delta T H^{3}}{\alpha \nu} .
$$




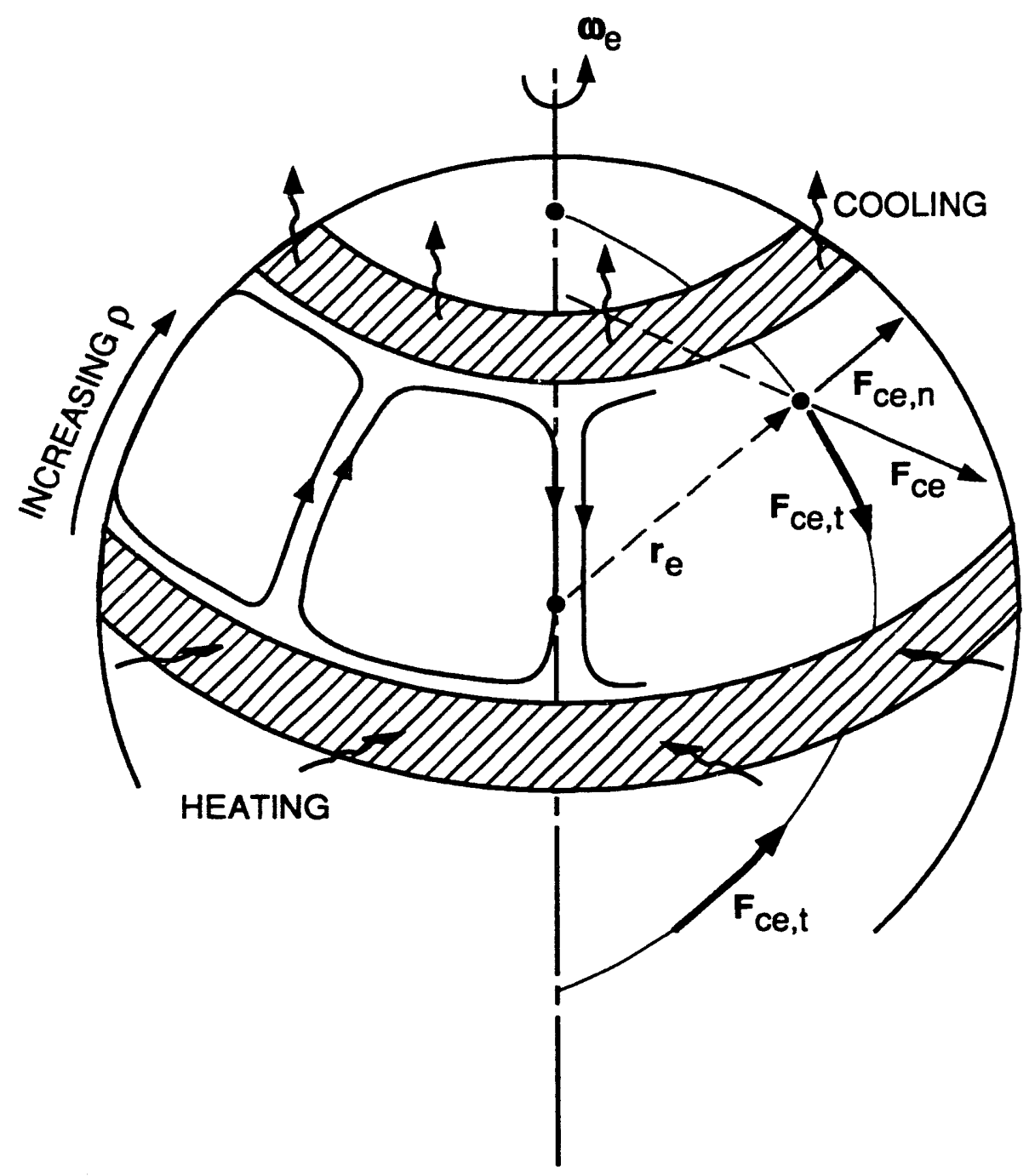

Fig. 3. Ocean circulation pattern illustrating analogy to convection cells in pool heated from below.

Because of the cubic dependence of $\mathrm{Ra}$ on the characteristic length, the value of $\mathrm{Ra}_{0}$ is extremely large, $\sim 10^{30}$. Compared with a critical value of the Rayleigh parameter of $\sim 10^{3}$ for the shallow pool, it is apparent that the large value of the analogous parameter, $R a_{0}$, is indicative of instability. 


\section{CIRCULATION MODEL TEST}

The rigorous method for testing the stability of a liquid with an imposed temperature and force field is to assume some set of perturbations and solve the dynamic equations for the perturbations. Decay with time is indicative of stability. Such a procedure would be quite complex for this case because of the presence of the free surface. A less rigorous alternative involves empirical examination of the result of the instability for consistency with the presumed driving force. For the case shown in Fig. 2, it can be shown that the flow resistance and the observed circulation rate are consistent with the observed temperature difference driving the convection. A similar consistency test may be applied to the case of liquid on a rotating sphere after recognizing that the effect of density variations on the tangential component of the centrifugal force provides an analogous driving force for circulation.

Figure 4 illustrates an idealized view of an observed oceanic circulation loop. Referring to Eq. (5), the tangential component of the centrifugal force on a volume element of liquid within the circulation can be written as

$$
d F_{c e, s}=\rho\left(\boldsymbol{r}_{e}\right) \omega_{e}^{2} R \cos \theta \sin \theta d V,
$$

where $\rho\left(r_{e}\right)$ is now a function of location on the sphere surface and $d V$ the volume element. The sense of $\mathrm{dF}_{\mathrm{ce}, \mathrm{t}}$ is toward the equator at all locations. The net effect of the tangential component of the centrifugal force is obtained by integrating around the flow path,

$$
F=\oint d F_{c e s,}\left(r_{e}\right)
$$

For the case of constant density, integration along the two north-south segments of the flow path in the direction of the flow yields equal and opposite contributions to the integral and thus a zero net effect. However, the density of the eastern, south-flowing leg emanating from the arctic is higher than the western leg. In this case, integration around the flow circuit is not zero due to the greater contribution of the eastern, colder leg of the path. We may obtain an estimate for the net circulating force $F$ using the following approximations: (1) a symmetric flow path (i.e., identical north-south flow segments), (2) negligible 


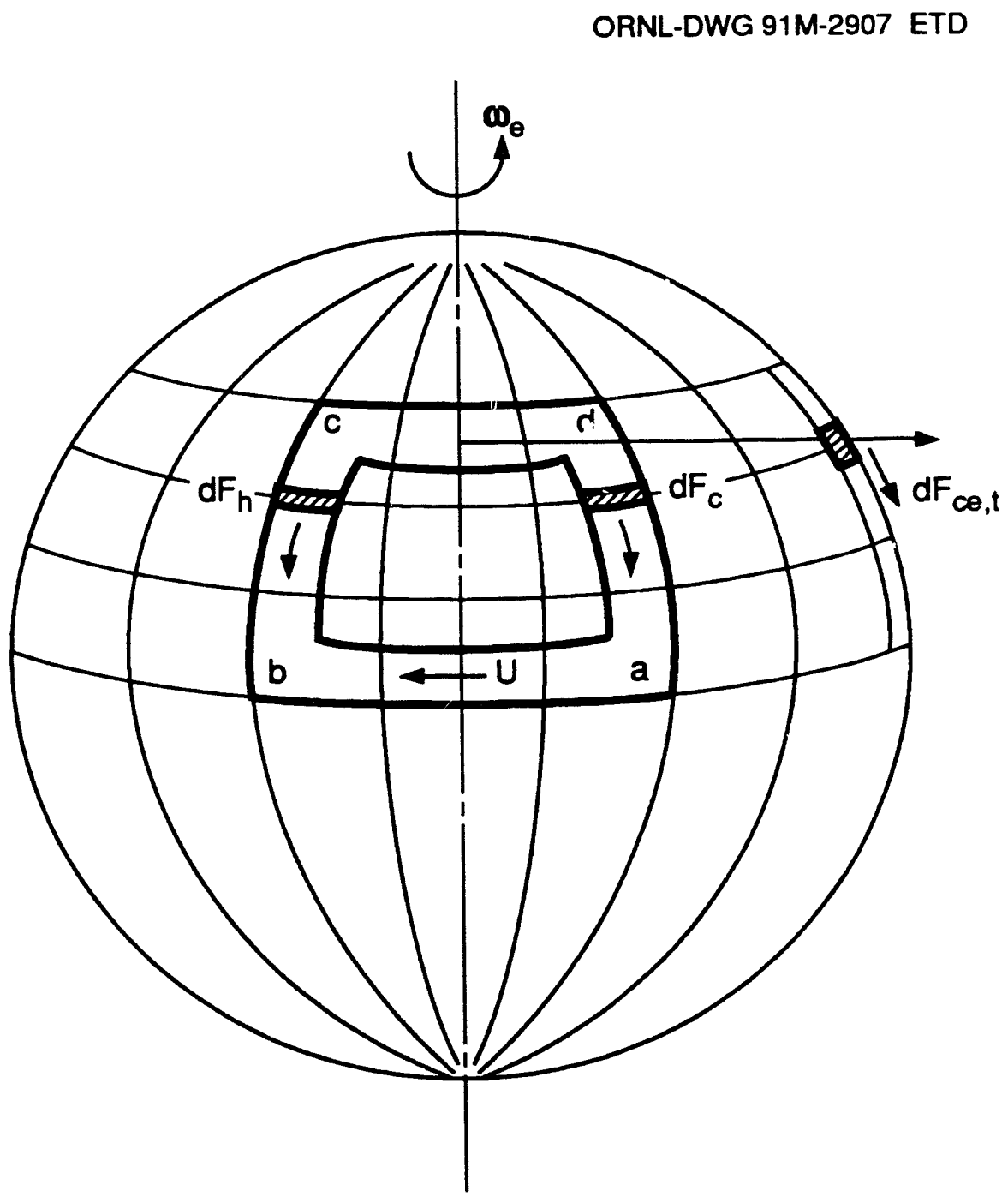

Fig. 4. Idealized oceanic circulation flow path.

contribution to the integral from the two segments of the path along the northern and southern lines of constant latitude, (3) a constant cross-sectional flow area A, and (4) average water densities $\rho_{\mathrm{h}}$ along the warmer leg flowing north and $\rho_{\mathrm{c}}$ along the cooler leg flowing south. Hence, the tangential component of the centrifugal force on a volume element of flow along the colder leg may be written,

$$
d F_{c}=\rho_{c} \omega^{2} R^{2} A \sin \theta \cos \theta d \theta \text {. }
$$


An identical relationship for the tangential force in the warm segment $\mathrm{dF}_{\mathrm{b}}$ may be written by substituting $\rho_{\mathrm{b}}$ for $\rho_{\mathrm{c}}$. These assumptions yield the net force for circulation due to the tangential component of the centrifugal force by integration of Eq. (10) along the flow path,

$$
F=\frac{1}{2} \Delta p \omega_{e}^{2} R^{2} A\left(\sin ^{2} \theta_{2}-\sin ^{2} \theta_{1}\right),
$$

where $\theta_{1}$ and $\theta_{2}$ are, respectively, the latitudes of the southern and northern segments of the flow circuit, and $\Delta \rho$ is the difference between $\rho_{c}$ and $\rho_{\mathrm{h}}$.

The magnitude of the driving force $F$, when equated to the velocity-dependent flow resistance, yields an expression for the velocity. An estimate of the flow resistance may be obtained by presuming the friction loss of the ocean current to be similar to that experienced by the turbulent flow of water in a conduit. For this case Lapple (1954) gives a simple approximation that the frictional loss expressed in terms of numbers of velocity heads, where one velocity head is the amount of kinetic energy per unit volume, given by $\rho U^{2} / 2$. According to Lapple, high Reynolds number flows experience about one velocity head friction loss in a distance equal to $\mathbf{4 0}$ conduit diameters. The total flow resistance is thus given by the product of the friction loss, expressed in terms of an unknown $\mathrm{K}$ number of velocity heads, and the flow area; that is,

$$
F_{R}=\frac{1}{2} K \rho A D^{2}
$$

where $U$ is the average velocity. An expression for $U$ is obtained by equating the driving force estimate $F$ with the estimated flow resistance $F_{R}$,

$$
U=\omega_{R} R \frac{\left[\Delta \rho\left(\sin ^{2} \theta_{1}-\sin ^{2} \theta_{2}\right)\right]^{\frac{1}{2}}}{R \rho} .
$$

We can obtain an estimate for $U$ from Eq. (14) using parameters representative of Gulf Stream flow. The mean latitudes of the southern and northern flow segments are approximately $15^{\circ} \mathrm{N}$ and $38^{\circ} \mathrm{N}$, respectively, according to current patterns developed by Sverdrup et al. (1942). We will assume the average temperatures of the northern and 
southern extremities of the flow to be 4 and $25^{\circ} \mathrm{C}$, respectively, which is equivalent to a density difference of $0.003191 \mathrm{~g} / \mathrm{cm}^{3}$. Since $\Delta \rho$ represents the difference between the average densities of the northerly and southerly segments, its value will be less by approximately a factor of 2 (i.e., $\Delta \rho=0.00159 \mathrm{~g} / \mathrm{cm}^{3}$ ). To obtain an estimate for $\mathrm{K}$, the length to the flow circuit and the dimensions of the flow cross section are required. The length of the flow circuit encompasses about $18,000 \mathrm{~km}$. According to Okubo and Pritchard (1969), the average width of the current is $150 \mathrm{~km}$ based on numerous observations. They indicate that there is a gradual decrease of velocity with depth so that there is no clear value for the depth of the current. For the purpose of this order-of-magnitude estimate, we will assume a current depth of $1 \mathrm{~km}$, at which point the velocity drops to $10 \mathrm{~cm} / \mathrm{s}$ compared with a maximum surface velocity of $150 \mathrm{~cm} / \mathrm{s}$. Okubo and Pritchard give the average volumetric flow adjacent to Chesapeake Bay as $70 \times 10^{6} \mathrm{~m}^{3} / \mathrm{s}$.

These parameter estimates give the following values: an effective diameter of flow (i.e., the hydraulic diameter that is equal to four times the flow area divided by the wetted perimeter) of $3.95 \mathrm{~km}$; therefore, a length-to-diameter ratio of $18,000 / 3.95$ or 4560 and number of velocity heads $\mathrm{K}$ of about 114 . The flow area, based on the arbitrary selection of the depth, is $150 \mathrm{~km}^{2}$, which yields an average current velocity of $47 \mathrm{~cm} / \mathrm{s}$ using the given value of the volumetric flow.

In comparison, substituting the cited parameters into Eq. (14) yields a value of $U=103$ $\mathrm{cm} / \mathrm{s}$ for the average current velocity. This favorable comparison indicates that the proposed mechanism generates sufficient force to account for at least a significant portion of the observed oceanic circulation.

\section{CLOSURE}

The proposed model suggests that a significant motive force for the major oceanic circulations results from the tangential component of the centrifugal body force acting upon water-density variations along the flow path. An expression for the mean current velocity based on this model is obtained using a highly idealized parameterization of the flow and a

fairly crude approximation of the flow resistance. For the velocity averaged over the flow cross section, substitution of Gulf Stream characteristics into the model yields a value of 103 $\mathrm{cm} / \mathrm{s}$, which compares sufficiently well with observations to indicate a significant effect. 
Further, it has been suggested that the oceanic circulations may be viewed as the hydrodynamically stable condition relative to that of a nnncirculating ocean in which heat is transported from equator to poles by means of molecular conduction. An analogous situation is the case of the shallow pool heated from below, which converts from a stationary condition to an eddy field above a critical Rayleigh number. For ocean currents, the tangential component of the centrifugal force plays a role analogous to that of the gravity body force in a shallow pool.

The model contains no mechanism for determination of flow direction. The wind shear may perhaps be considered as the causative mechanism for perturbation of the initial stationary state and thereby would fix the direction of rotation. In such case, the circulatory effects of the centrifugal body force, wind shear, and the Coriolis force would be additive.

If the concepts presented in this report prove to be valid, a more accurate evaluation of the net circulation force F, as given by Eqs. (9) and (10), would be in order. A more detailed arcount of variation of liquid density and flow cross-sectional area with latitude would replace the approximation represented by Eq. (12). In addition, the crude approximation for the circulatory flow resistance, given by Eq. (13), should be replaced by more appropriate estimates based on eddy interactions between the current and the slower surrounding liquid. Mean values of products of velocity fluctuation from the mean, such as reported by Dewar and Bane (1989), are the type of data required for improved estimation of the flow resistance.

Several considerations indicate interesting lines for further study. For example, it may be noted that seasonal variations in the rate of oceanic circulation due to $F_{c e}$ would differ from that predicted by wind-driven models. The centrifugal body force reaches maximum during the period of maximum density difference between the two north-south legs of the circulation. Since the period of greatest insolation difference between equatorial and arctic regions occurs in winter, the period of maximal $F_{c e}$ would therefore lag in the winter months by a period that depends on the thermal inertia of the current. In contrast, data summarized by Boning et al. (1991) indicate that the westerly directed wind stresses in equatorial regions and easterly directed wind stresses at about $50^{\circ} \mathrm{N}$ both peak during the winter months. Therefore, wind-driven models should show peak acceleration in the winter. Observation of the time of maximum flow acceleration would therefore yield information on the relative effects of the centrifugal force and wind-shear mechanisms. 
In addition, an accurate analysis of the stability criterion for oceanic flows would be informative. The method would follow that used by Rayleigh for shallow pools but would entail the additional complexity due to the presence of the free surface. The result would yield a critical oceanic Rayleigh number above which the stationary condition is unstable relative to circulation and replace the intuitive expression in Eq. (8). 


\section{REFERENCES}

A. Bejan (1984), Convection Heat Transfer, John Wiley and Sons, Chapters 5 and 11.

C. W. Boning et al. (1991), "Monthly Mean Wind Stress and Sverdrup Transports in the North Atlantic," Journal of Phys. Oceanography 21, 221-235.

K Bryan (1969), "A Numerical Method for the Study of the Circulation of the World Ocean," Journal of Comp. Phys. 4, 347-376.

W. K. Dewar and J. M. Bane (1989), "Gulf Stream Dynamics. Part 1: Mean Flow Dynamics at $73^{\circ} \mathrm{W},{ }^{n}$ Journal of Phys. Oceanography 19, 1559-1573.

C. Eckart (1962), "The Equations of Motion of Sea-Water," in The Sea, Vol. 1, pp. 31-41, John Wiley and Sons, New York.

N. P. Fofonoff (1962), "Dynamics of Ocean Current," in The Sea, Vol. 1, pp. 323-395, John Wiley and Sons, New York.

W. R. Holland (1977), "Oceanic General Circulation Models," in The Sea, Vol. 6, p. 3, John Wiley and Sons, New York.

W. R. Holland and J. C. McWilliams (1987), "Computer Modeling in Physical Oceanography from Global Circulation to Turbulence," Phys. Today, 51-57 (October 1987).

C. E. Lapple (1954), Fluid and Paricle Mechanics, University of Delaware, pp. 32-37.

A. Okubo and D. W. Pritchard (1969), Processes of Mixing in the Ocean and Coastal Waters, Chesapeake Bay Institute Report NYO-3190-40.

Rayleigh (1916), "On Convection Currents in a Horizontal Layer of Fluid When the Higher Temperature is on the Underside," Philos. Mag. 6, 529-546.

R. W. Stewart (1969), "The Atmosphere and the Ocean," Science American (September 1969).

H. V. Sverdrup (1942), The Oceans, Prentiss-Hall, New York, p. 1087. 
ORNL-6692

Distribution Category Uc-262

\section{INTERNAL DISTRIBUTION}

1. V. Alexiades

2. W. G. Craddick

3. J. B. Drake

4. M. Fontana

5. E. C. Fox

6. Uri Gat

7. R. L. Graves

8. J. E. Jones Jr.

9. R. L. Jolly

10. L. Jung

11. H. T. Kerr

12. T. S. Kress

13. R. M. Schilling

14. M. Siman-Tov
15. W. B. Snyder, Jr.

16. D. G. Thomas

17. M. L. Tobias

18. A. W. Trivelpiece

19. E. W. Whitfield

20-34. R. P. Wichner

35. G. L. Yoder, Jr.

36. A. Zucker

37. Central Research Library

38. Document Reference Section

39-40. Laboratory Records Department

41. Laboratory Records, ORNL RC

42. ORNL Patent Section

\section{EXTERNAL DISTRIBUTION}

43. Office of Assistant Manager for Energy Research and Development, DOE Field Office, Oak Ridge, P.O. Box 2008, Oak Ridge, TN 37831-6269

44-45. P. R. Gent, National Center for Atmospheric Research, P.O. Box 3000, Boulder, CO 80307-3000

46. R. Chervin, National Center for Atmospheric Research, P.O. Box 3000, Boulder, CO 80307

47. W. Dannevik, Lawrence Livermore Laboratory, P.O. Box 808, L16, Livermore, CA 94550

48-59. Given distribution as shown in DOE/OSTI-4500-R75 under Category UC-262 (Ocean Energy Systems) 

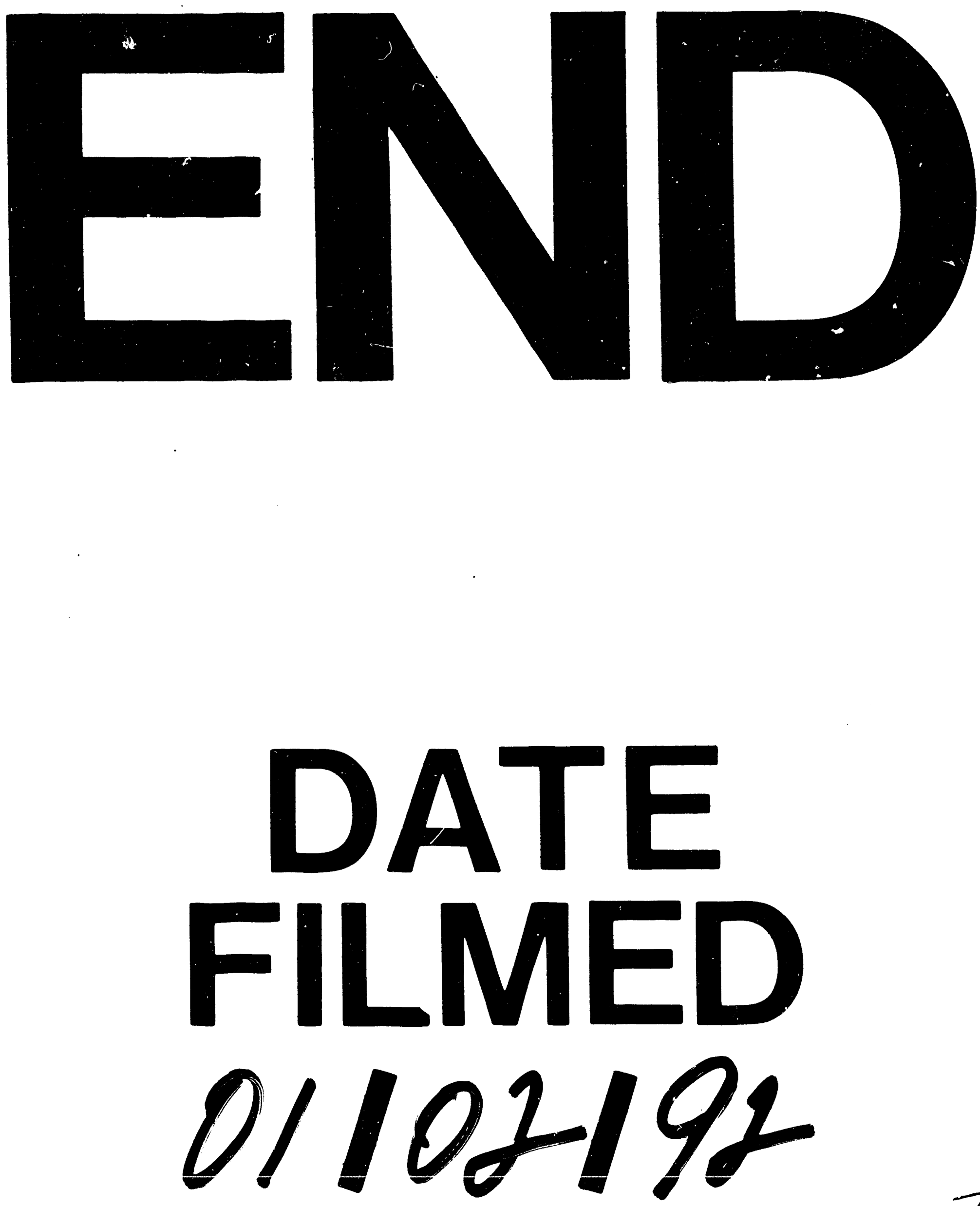
